Supporting Information

\title{
Toward the Synthesis of Sub-15 nm Ag Nanocubes with Sharp Corners and Edges: The Roles of Heterogeneous Nucleation and Surface Capping
}

\author{
Aleksey Ruditskiy ${ }^{\dagger}$ and Younan Xia ${ }^{\dagger, \S, * ; *}$
}

${ }^{\dagger}$ School of Chemistry and Biochemistry, Georgia Institute of Technology, Atlanta, Georgia 30332, United States

${ }^{\S}$ The Wallace H. Coulter Department of Biomedical Engineering, Georgia Institute of Technology and Emory University, Atlanta, Georgia 30332, United States

${ }^{\star}$ School of Chemical and Biomolecular Engineering, Georgia Institute of Technology, Atlanta, Georgia 30332, United States

*Corresponding author: younan.xia@bme.gatech.edu 


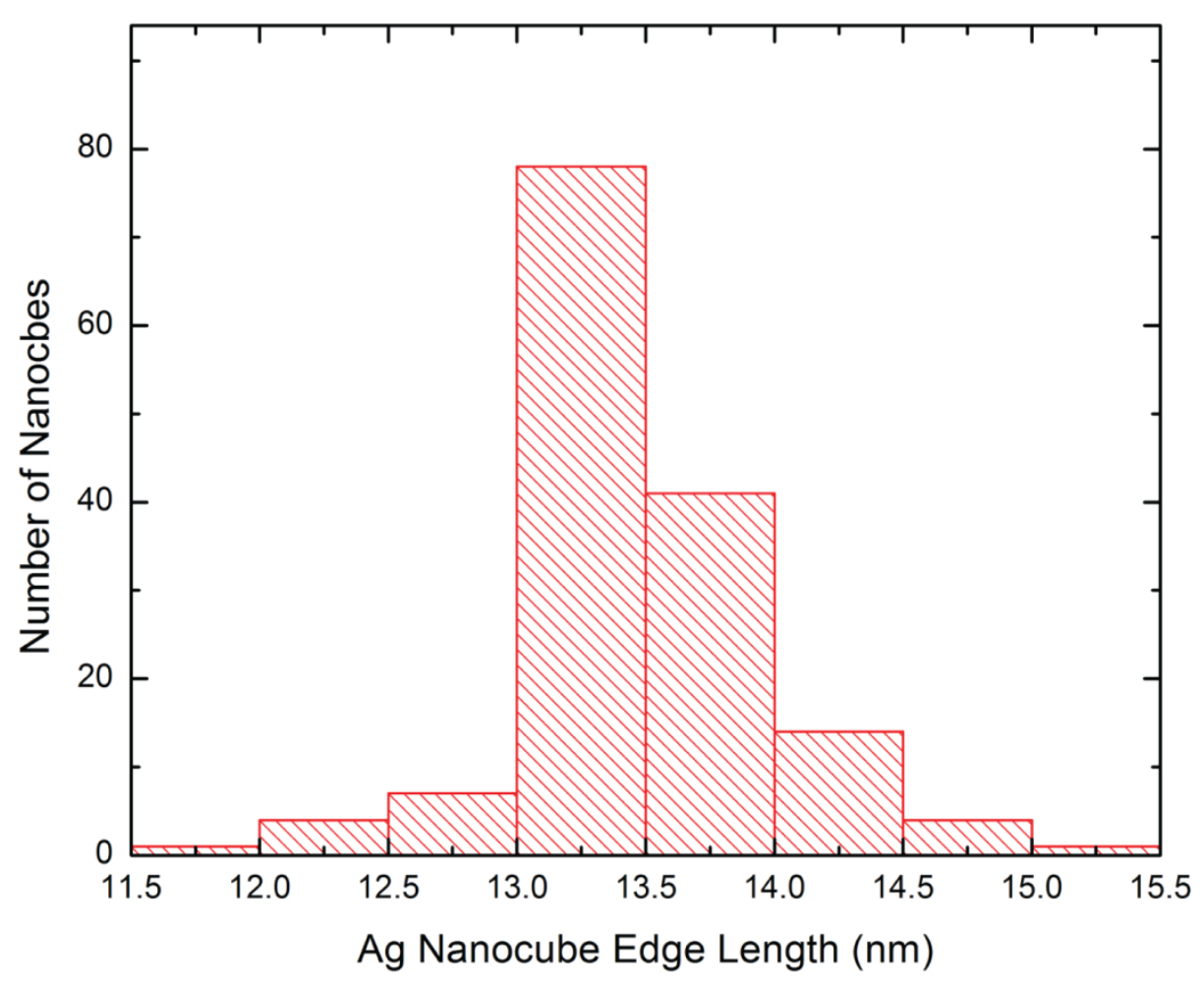

Figure S1. A histogram showing the edge length distribution of Ag nanocubes shown in Figure $1 \mathrm{~A}$. 


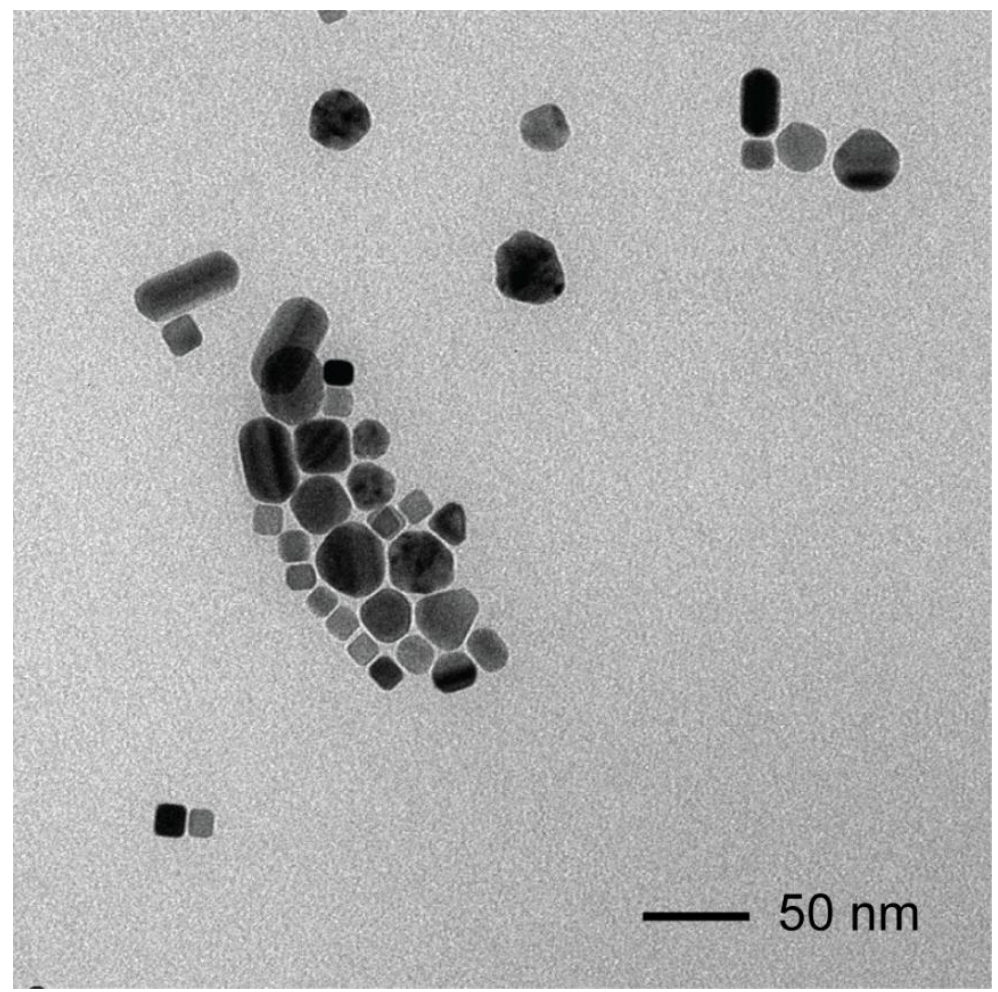

Figure S2. TEM image of the reaction product synthesized with a delay time of $1 \mathrm{~min}$, while all other parameters were kept the same as in the standard synthesis. 

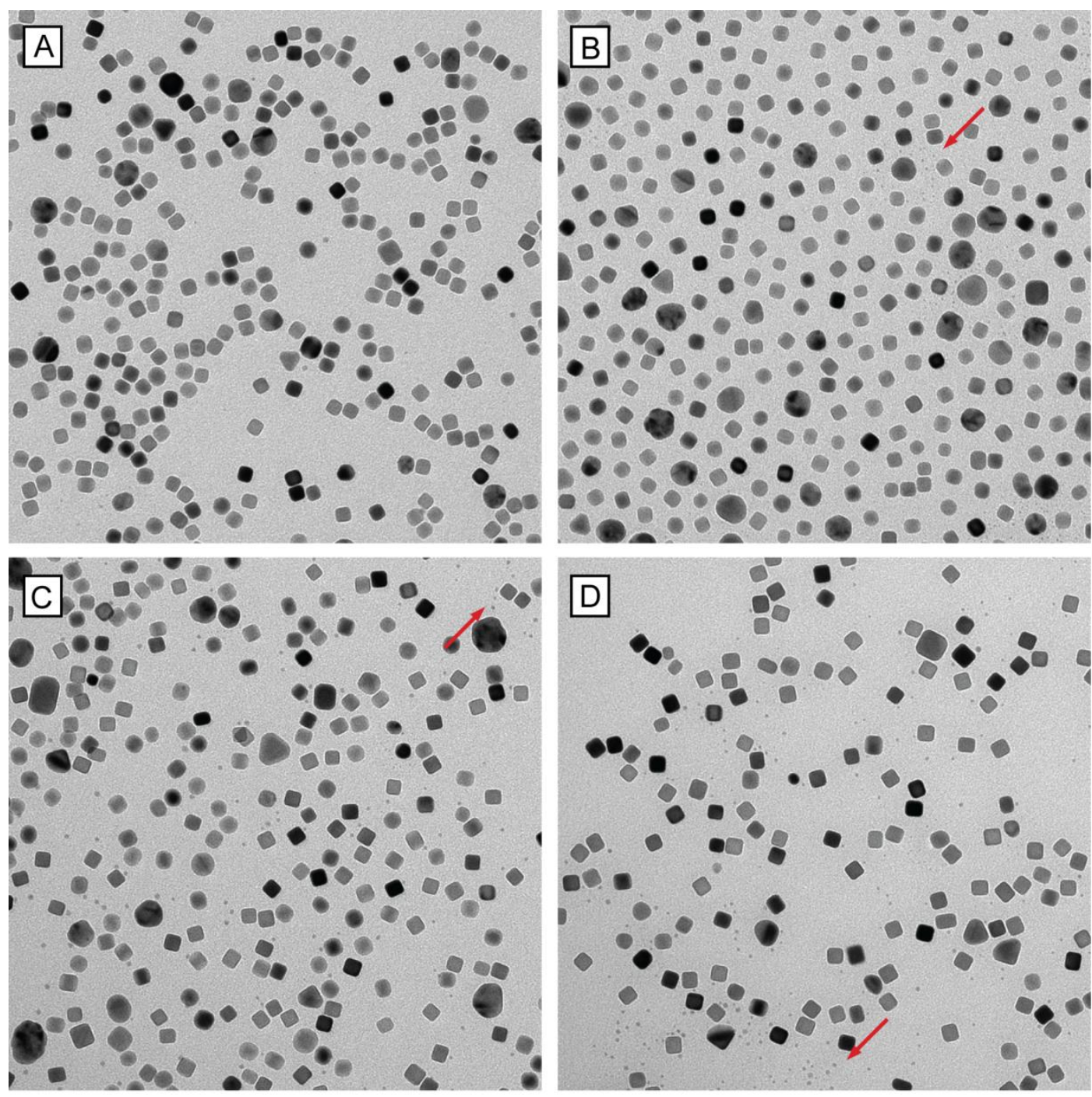

$50 \mathrm{~nm}$

Figure S3. TEM images of reaction products obtained with prolonged growth times: (A) 15, (B) 30, (C) 45, and (D) $60 \mathrm{~min}$, whereas all other parameters were kept the same as in the standard

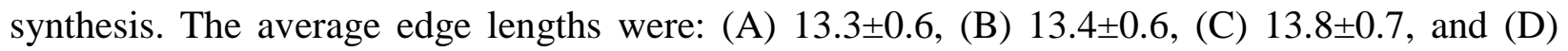
$14.3 \pm 0.6 \mathrm{~nm}$. Note the formation of sub-5 $\mathrm{nm}$ particles, as denoted by red arrows. 


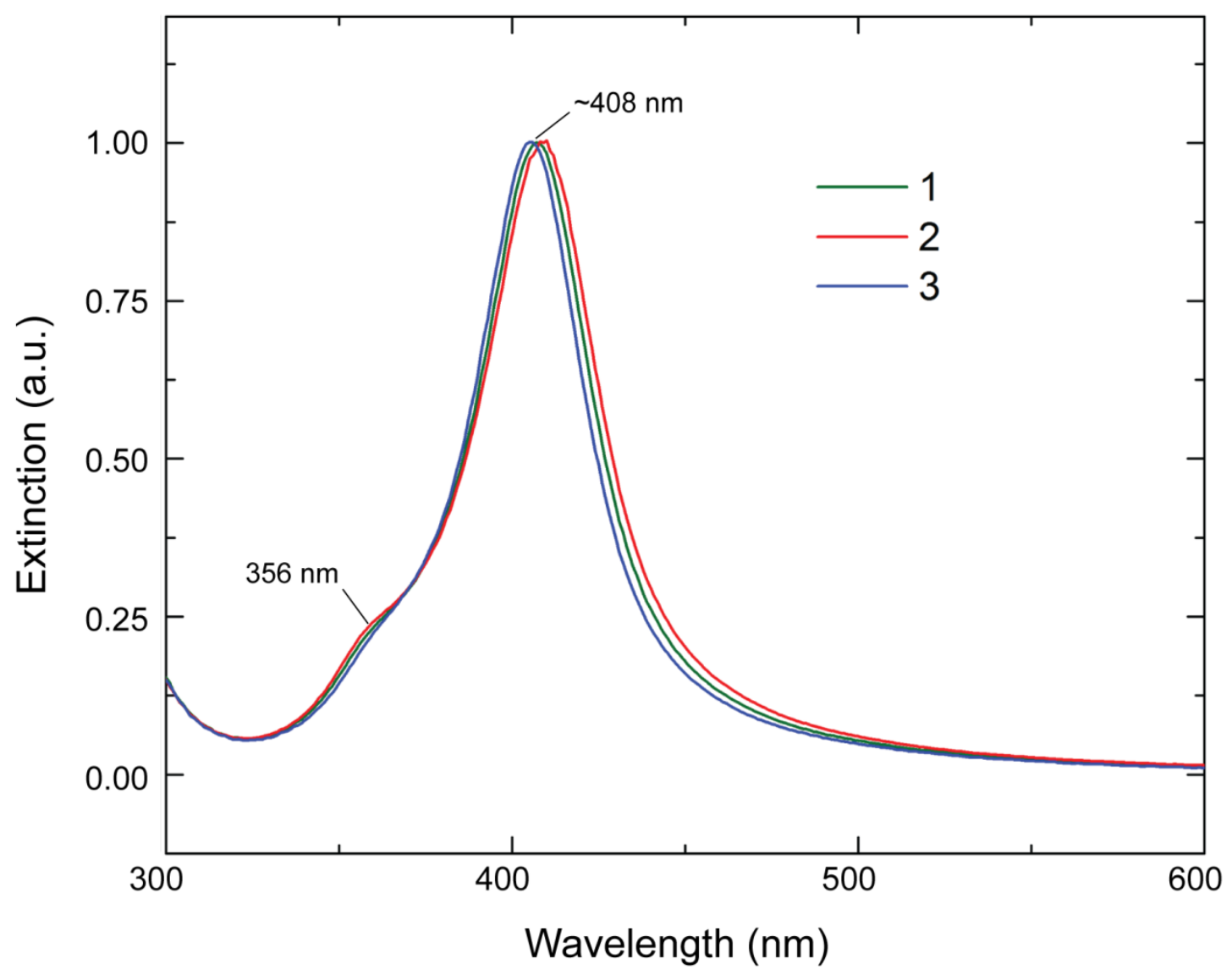

Figure S4. UV-Vis spectra of three different batches of 13-nm Ag nanocubes, as a demonstration of reproducibility for standard synthesis. 


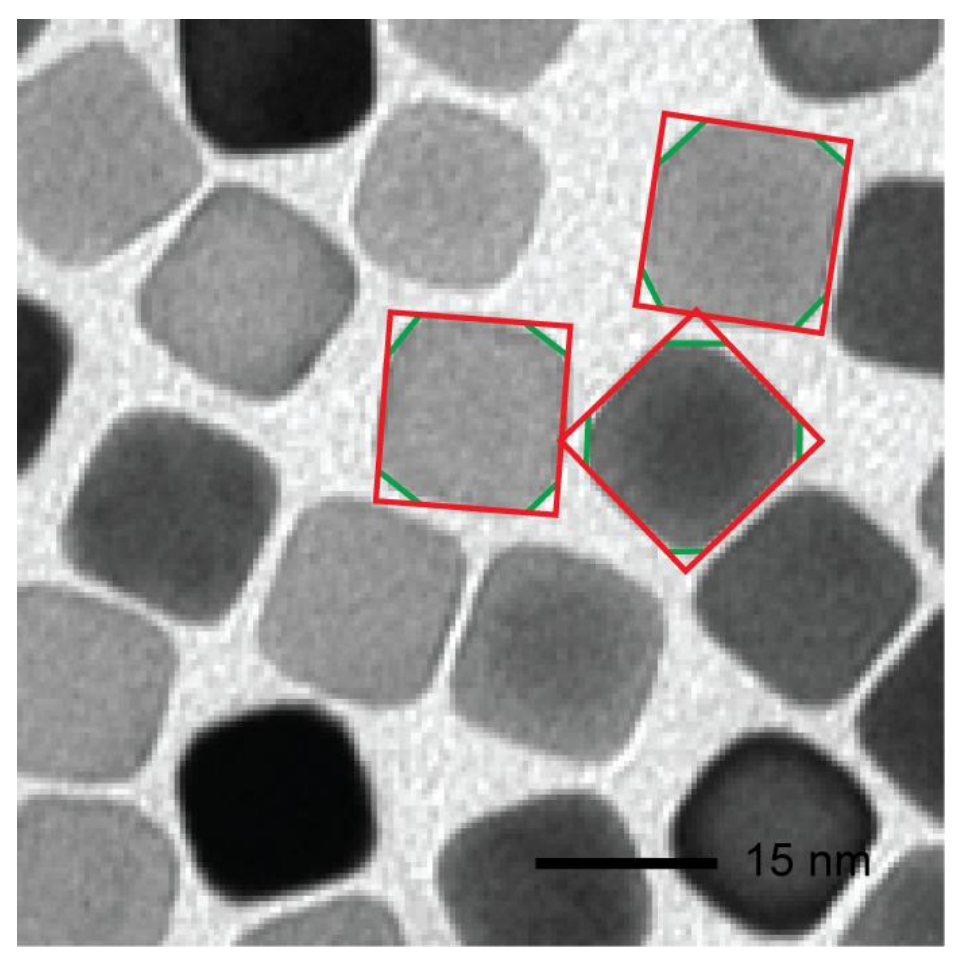

Figure S5. Illustration showing how TEM imaging is used to determine the corner/edge sharpness of the 15-nm Ag nanocubes. The fitted square outline is denoted in red. The 2-D area of the Ag nanocube is determined by manually subtracting the triangular areas of the missing corners, marked in green, from the area of the fitted square outline. The measure of corner/edge sharpness is defined as the ratio between the area occupied by a Ag nanocube and the area of the fitted square outline. We obtained an average value by analyzing 100 randomly selected nanocubes. 

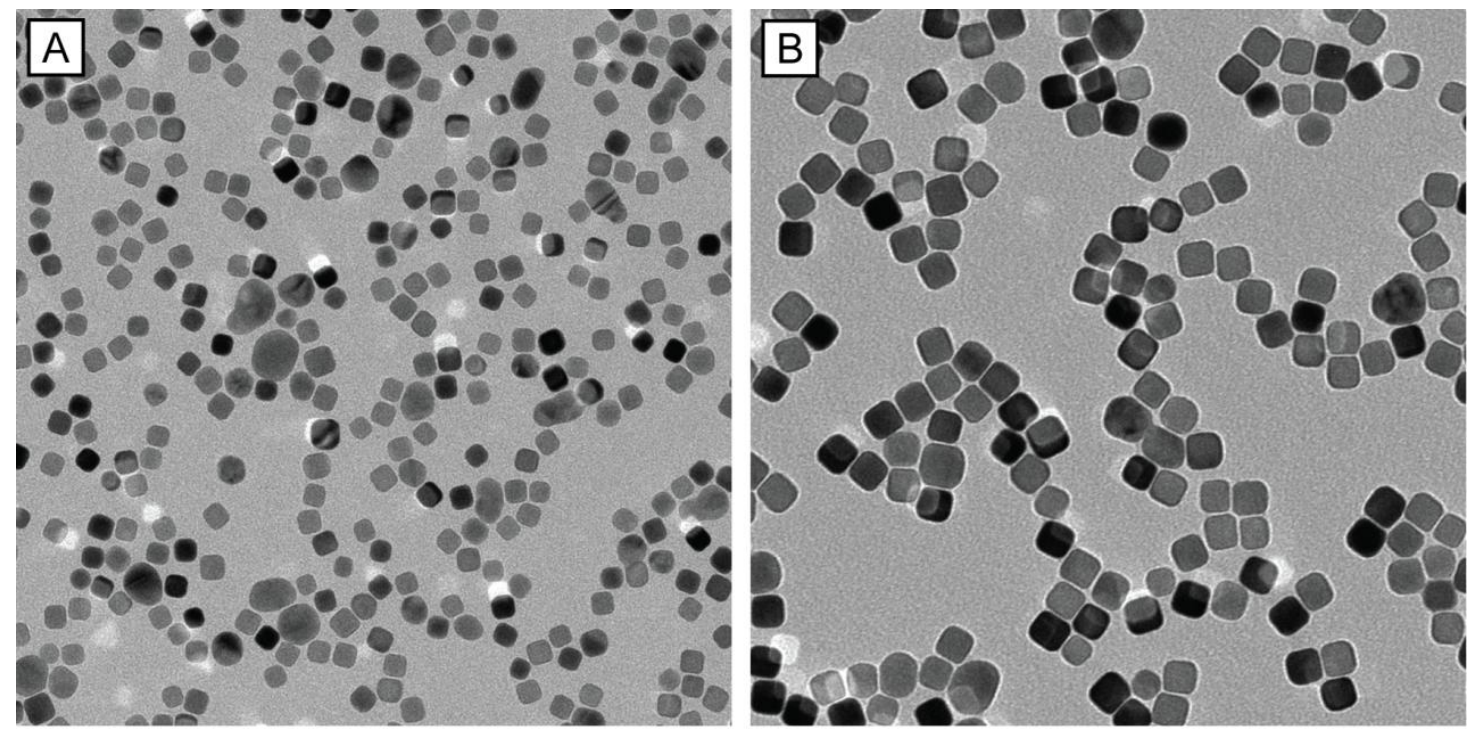

$50 \mathrm{~nm}$

Figure S6. TEM images of $\mathrm{Ag}$ nanocubes produced by substituting the $\mathrm{NaHS} \cdot 1.5 \mathrm{H}_{2} \mathrm{O}$ used in the standard procedure with an equal molar concentration of $\mathrm{Na}_{2} \mathrm{~S} \cdot 9 \mathrm{H}_{2} \mathrm{O}$, with different delay times. The average edge lengths of the nanocubes were: (A) $12.7 \pm 0.5$ and (B) $18.1 \pm 0.7 \mathrm{~nm}$. The delay times used were: (A) 5 and (B) 25 min. 